\title{
Comparison of CD44 expression in primary tumours and metastases of colorectal cancer
}

\author{
R. BENDARDAF ${ }^{1,2}$, A. ALGARS ${ }^{1,2}$, A. ELZAGHEID ${ }^{1,3}$, E. KORKEILA ${ }^{1}$, R. RISTAMÄKI ${ }^{1}$, \\ H. LAMLUM ${ }^{1,2}$, Y. COLLAN ${ }^{3}$, K. SYRJÄNEN ${ }^{1}$ and S. PYRHÖNEN ${ }^{1}$ \\ ${ }^{1}$ Department of Oncology and Radiotherapy, Turku University Hospital, Savitehtaankatu 1, PB 52, FIN 20521; \\ ${ }^{2}$ Medicity Research Laboratory, University of Turku, Tykistökatu 6A; ${ }^{3}$ Department of Pathology, \\ University of Turku, Kiinamyllynkatu 10, FIN-20520, Turku, Finland
}

Received February 10, 2006; Accepted March 31, 2006

\begin{abstract}
A better understanding on the development of a metastatic phenotype in colorectal cancer (CRC) is essential to help identify patients at high risk for metastasis. Therefore, we have studied the role of the CD44 family of trans-membrane glycoprotein in the process of CRC metastasis, by examining the expression of CD44s and CD44v6 in primary tumours and their metastatic lesions in 46 patients using immunohistochemistry. The expression of both CD44s and CD44v6 was significantly higher (moderate/strong) in primary tumours as compared to their metastases $(\mathrm{p}=0.008$, $\mathrm{p}=0.0001$, respectively). CD $44 \mathrm{~s}$ expression in metastases increased with the degree of the histological grade $(\mathrm{p}=0.009)$ and invasiveness of the primary tumour $(\mathrm{p}=0.002)$. Diseasefree survival (DFS) was shorter in patients who had metastases with a strong/moderate expression of CD44s as compared to those with negative/weak expression (8.3 months vs 16.9 months $\mathrm{p}=0.221$, respectively). Our finding that CD44s expression in metastatic lesions may reflect the aggressiveness of the primary tumour from which it has originated implicates an important link between the two lesions. CD44 expression may also provide valuable biological information as suggested by the observation that upregulated CD44s expression in metastases is associated with a shorter DFS.
\end{abstract}

\section{Introduction}

Colorectal cancer (CRC) was the second most frequent cancer in Europe in 2004, responsible for 13\% $(376,400)$ of all

Correspondence to: Dr R. Bendardaf, Department of Oncology and Radiotherapy, Turku University Hospital, Savitehtaankatu 1, PB 52, FIN-20521, Turku, Finland

E-mail: riyben@utu.fi

Abbreviations: CRC, colorectal cancer; DFS, disease-free survival; DSS, disease-specific survival; CEA, carcinoembryonic antigen; ECM, extracellular matrix; BM, basement membrane; HA, hyaluronic acid; T, depth of primary tumour invasion; G, primary tumour grade

Key words: colorectal cancer, CD44, stage, grade, survival incident cancer cases. It is also the second most frequent cause of cancer mortality, with $11.9 \%(203,700)$ annual deaths (1). CRC has a relatively poor prognosis, the outcome being determined by the extent of local and metastatic tumour spread. The estimated 5-year survival rate ranges from nearly $90 \%$ in stage I disease (Dukes A) to $<10 \%$ in patients with metastatic disease (Dukes D) (2). Postoperative adjuvant chemotherapy is known to improve the outcome in stage III (Dukes stage C) CRC and is widely accepted as standard therapy (3). The majority (up to 90\%) of CRC patients undergo surgery, but $\sim 50 \%$ of those patients initially believed to be cured by surgery, will relapse and die of their disease $(4,5)$. Once metastasis has developed, treatment is mostly palliative. There is an urgent need to focus research on the molecular events involved in the process of metastasis. Identifying the molecular markers of a metastatic phenotype is a prerequisite for developing new therapeutic approaches in CRC.

CD44, a trans-membrane glycoprotein molecule, is a widely expressed cell surface hyaluronan receptor found in normal epithelial, mesenchymal and haematopoietic cells. This family of molecules is generated from a single gene by alternative splicing and differential glycosylation (6). The interaction between CD44 and hyaluronan mediates both cell-cell and cell-extracellular matrix interactions. The CD44 family is important in a variety of physiological and pathological processes, including wound healing and leukocyte extravasation at sites of inflammation, development of metastasis $(6,7)$. These biological properties are essential for the activity of normal cells, but they are also associated with many of the pathological functions of cancer cells (8). CD44 is known to induce tumour necrosis factor (TNF) and interleukin-1 (IL-1) release, and consequently, it is considered to play an important role in the metastatic process. However, the precise mechanisms of CD44 functions are still unknown $(9-11)$.

In normal colorectal mucosa, CD44s is only expressed by a few crypt epithelial cells, but is present in $>80 \%$ of the stromal cells (12). A potential role for CD44 in colorectal tumorigenesis was first suggested by the detection of CD44s in tumour tissues, shown to be markedly increased in adenomas and CRC (12). Similarly, the CD44v6 isoform has also been linked with CRC in several recent studies (12-14). 
Table I. Key characteristics of the patients and their tumours.

\begin{tabular}{ll}
\hline Variables & Number (\%) \\
\hline No. of patients & $46(100)$ \\
Female & $27(58.7)$ \\
Male & $19(41.3)$
\end{tabular}

Primary tumour (T)

$\begin{array}{cc}\text { T1 } & 0(0.0) \\ \text { T2 } & 1(2.2) \\ \text { T3 } & 37(80.4) \\ \text { T4 } & 8(17.4)\end{array}$

Nodal involvement $(\mathrm{N})$

NO

N1

$\mathrm{Nx}$

Tumour grade $(\mathrm{G})$

Grade 1

$11(23.9)$

Grade 2

Grade 3

Dukes staging

Stage B

9 (19.6)

Stage C

$10(21.7)$

Stage D

$27(58.7)$

Site of metastases

Liver

$14(30.4)$

Local

$6(13.0)$

Omentum

$6(13.0)$

Mesentry

$4(8.7)$

Ovary

Peritoneum

Lymph nodes

Lung

Bone

Other

$4(8.7)$

To elucidate the role of CD44 in the development of a metastatic phenotype in CRC, we examined the expression of CD44s and CD44v6 isoforms both in primary tumours and their metastatic lesions in a series of patients with locally advanced or metastatic CRC.

\section{Materials and methods}

Patients, treatment and follow-up. A series of 46 patients were treated for Dukes B, C and D colorectal cancer at the Department of Oncology and Radiotherapy, Turku University Hospital and six other hospitals of the same hospital district, between January 1996 and August 2003. The key characteristics of the patients and their tumours are summarised in Table I. At diagnosis, 27 patients had metastasis and the remaining patients developed a metastatic disease during the follow-up. The patients were prospectively followed-up until death, with a mean follow-up time of 32.3 months (median 28.2).

The collection of tumour samples was approved by the National Authority for Medicolegal Affairs. The study design was approved by the local Ethics Committee, and it was compliant with the Declaration of Helsinki.

Immunohistochemical detection of CD44s, and CD44v6 expression. Forty-six pairs of formalin-fixed, paraffinembedded primary tumours and their metastases were obtained from 46 patients. Sections were cut serially at $5 \mu \mathrm{m}$ for routine $\mathrm{H} \& \mathrm{E}$ staining and for immunohistochemical analysis. An experienced pathologist confirmed all histological diagnoses.

CD44s and CD44v6 expression was studied immunohistochemically using two monoclonal antibodies, Hermes 3 and 20E6. Hermes 3 recognizes an epitope in the constant region of human $\mathrm{CD} 44$, while the 20E6 antibody recognizes the v6-isoform of the CD44 molecule. Production and characterization of these $\mathrm{mAbs}$ has been described previously $(7,15)$. Detection was performed using the streptavidin-biotin method (Vectastain ABC kit). Formalin-fixed paraffinembedded sections were deparaffinised in xylene, rehydrated in graded alcohol, immersed in $0.01 \mathrm{M}$ citrate buffer ( $\mathrm{pH}$ 6.0), heated in a domestic microwave oven at full power for $2 \times 7 \mathrm{~min}$ and left in the buffer to cool to room temperature. The sections were incubated in $0.3 \%$ hydrogen peroxide for $20 \mathrm{~min}$ to block endogenous peroxidase activity. Incubation with the primary antibody diluted in $1 \%$ bovine serum albumin/Trisbuffered $\mathrm{NaCl}(\mathrm{CD} 44 \mathrm{~s} \mathrm{1:100}$ and $\mathrm{CD} 44 \mathrm{v} 6$ 1:30) was carried out overnight in a humid chamber at $4^{\circ} \mathrm{C}$. The following day the slides were washed and incubated first with the biotinylated secondary antibody $\left(30 \mathrm{~min}, 20^{\circ} \mathrm{C}\right)$, then with avidin-biotinperoxidase complex $\left(30 \mathrm{~min}, 20^{\circ} \mathrm{C}\right)$. Positive staining was visualised with 3,3' diaminobenzidine (DAB) substrate solution and the sections were counterstained with Mayer's haematoxylin. As negative controls, slides were processed with the omission of the primary antibody.

CD44s and CD44v6 expression was assessed by two independent observers blinded to the clinical data (R.B. and H.L.). The expression was weighted according to the total tumour area and categorised according to its intensity, following the method described in detail previously (16).

Statistical analysis. Statistical analyses were performed using the SPSS ${ }^{\circledR}$ (SPSS, Inc., Chicago, USA) and STATA (Stata Corp., TX, USA) software packages (SPSS for Windows, version 12.0.1 and STATA/SE 9.1). Frequency tables were analysed using the Chi-square test, with the likelihood ratio (LR) or Fisher's exact test being used to assess the significance of the correlation between the categorical variables. Differences in the means of continuous variables were analysed using non-parametric tests (Mann-Whitney) or Kruskall-Wallis for 2- and K-independent samples, respectively. ANOVA (analysis of variance) was only used for deriving the mean values (and the SD) of each individual category. Univariate survival (life-table) analysis for the outcome measure (DSS, DFS) was based on Kaplan-Meier method. Multivariate survival analysis was run by using Cox's proportional hazards model in a backward stepwise manner 

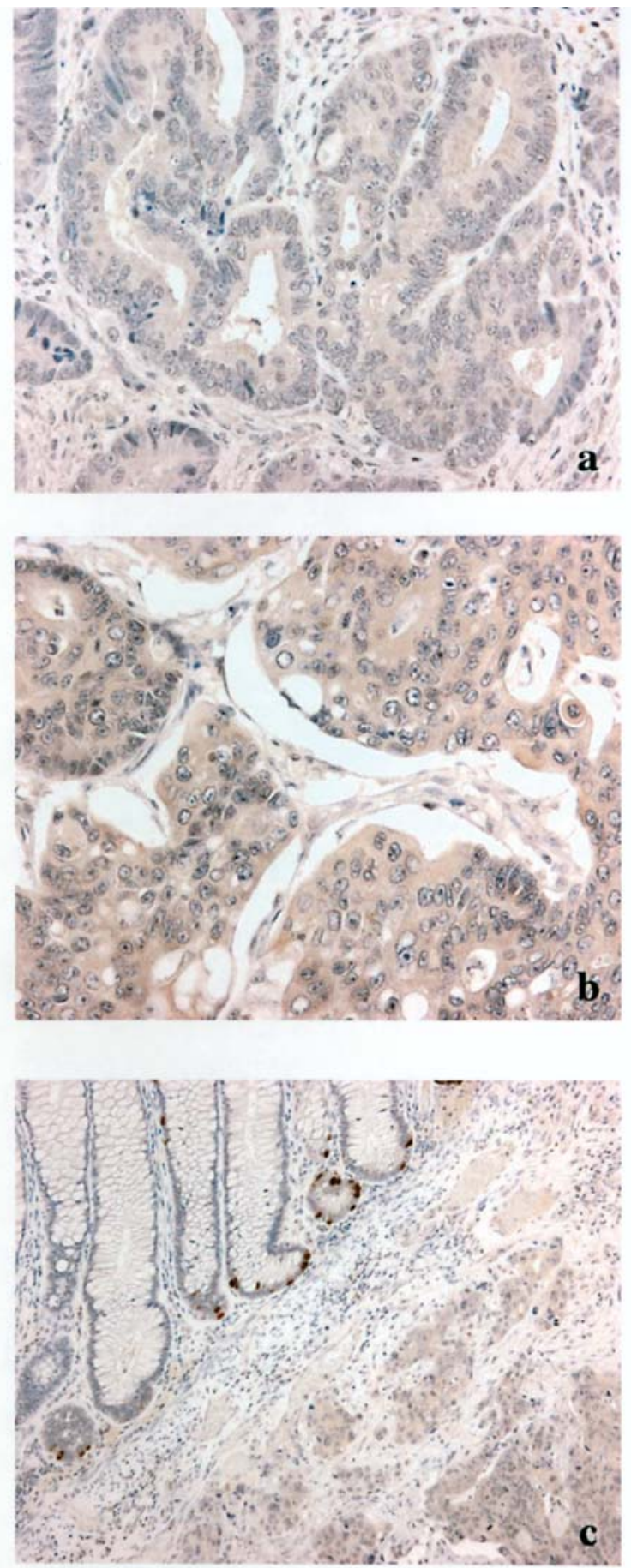
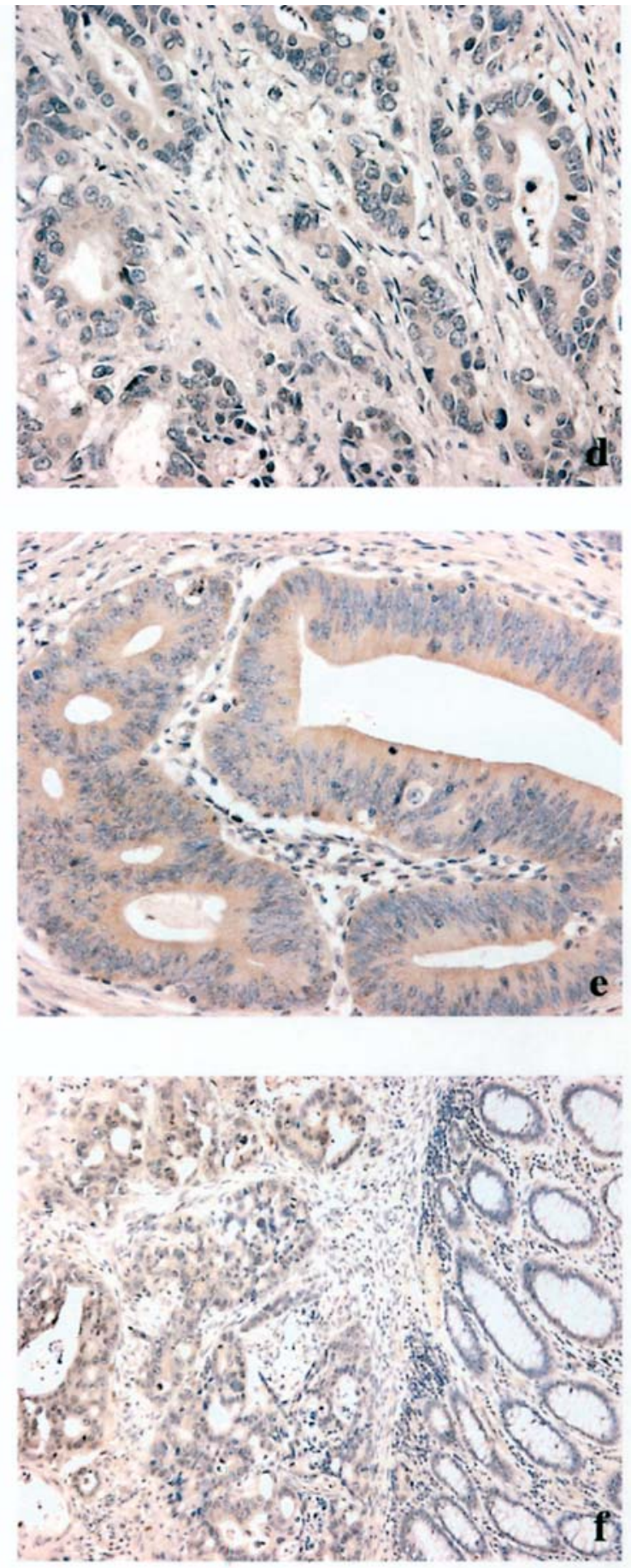

Figure 1. Immunohistochemical staining of CD44s (a-c) and CD44v6 (d-f); (a and d) weak expression, (b and e) strong expression (x20 magnification), (c and f) comparison of normal and tumour tissue; (c) CD44s expression can be seen in normal epithelial cells at the base of crypts (x10 magnification).

with the log-likelihood ratio (L-R) significance test, and using the default values for entry and exclusion criteria. The assumption of proportional hazards was controlled by logminus-log (LML) survival plots. In all tests, the values $\mathrm{p}<0.05$ were regarded statistically significant.

\section{Results}

The expression of both CD44s and CD44v6 was scored as negative, weak, moderate and strong, and for statistical analysis categorised into two groups; negative/weak or moderate/ strong expression, as illustrated in Fig. 1.
None of the primary tumours showed negative expression of CD44s, while 20 cases showed weak expression, 20 demonstrated moderate expression and the remaining 6 had strong expression. Comparing the primary tumours with their metastases, the latter showed absent or weak expression more often ( $p=0.008$, Fisher's exact test) (Fig. 2a). CD44v6 expression showed a similar pattern, with a more pronounced difference between the primary tumours and the metastases $(\mathrm{p}=0.0001)$ (Fig. 2b).

CD44s (but not CD44v6) expression in metastases significantly increased with the degree of primary tumour invasion $(\mathrm{T})(\mathrm{p}=0.002$, Fisher's exact test). Twenty-seven 
A

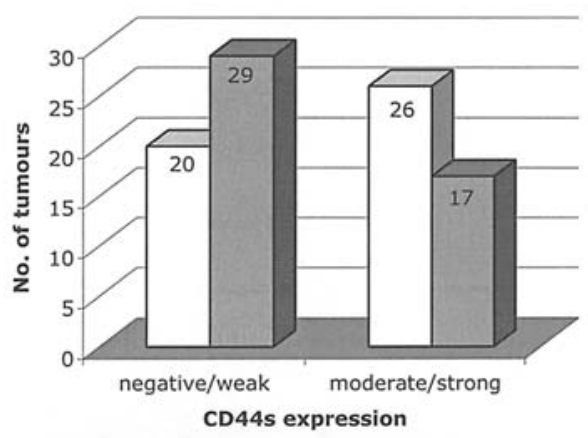

B

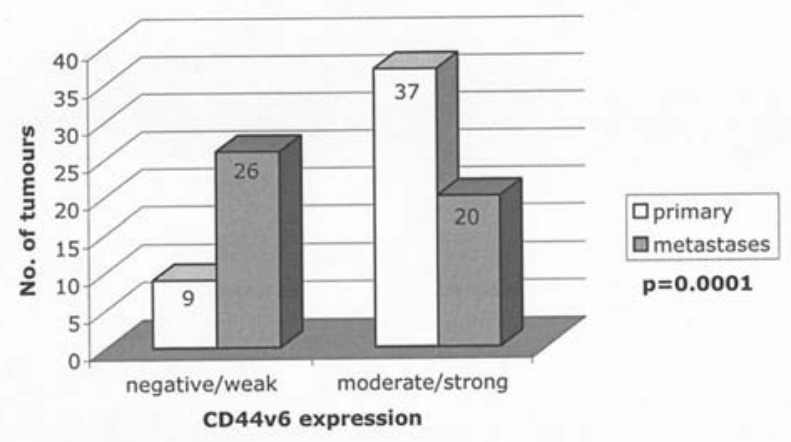

Figure 2. (A) Comparison of CD44s expression in primary and metastatic lesions. (B) Comparison of CD44v6 expression in primary and metastatic lesions.

Table II. CD44s expression in metastases compared to the primary tumour stage and grade.

\begin{tabular}{cccc}
\hline \multicolumn{4}{c}{$\begin{array}{c}\text { CD44s expression in } \\
\text { metastases }\end{array}$} \\
\cline { 2 - 3 } & $\begin{array}{c}\text { Negative/ } \\
\text { weak }\end{array}$ & $\begin{array}{c}\text { Moderate/ } \\
\text { strong }\end{array}$ & \\
\hline Primary tumour stage & & & $\mathrm{p}=0.002^{\mathrm{a}}$ \\
T2 & $1(3.4)$ & $0(0)$ & \\
T3 & $27(93.1)$ & $10(58.8)$ & \\
T4 & $1(3.4)$ & $7(41.2)$ & \\
Tumour grade & & & $\mathrm{p}=0.009^{\mathrm{a}}$ \\
Grade 1 & $10(34.5)$ & $1(5.9)$ & \\
Grade 2 & $18(62.1)$ & $11(64.7)$ & \\
Grade 3 & $1(3.4)$ & $5(29.4)$ & \\
\hline
\end{tabular}

${ }^{\mathrm{a}}$ Fisher's exact test.

percent of all $\mathrm{T} 3$ tumours had metastases with moderate to strong CD44s expression, whereas $87.5 \%$ of metastases from T4 tumours showed moderate to strong CD44s expression (Table II). Expression of CD44s in metastases was also associated with the primary tumour grade, increasing with the loss of differentiation (i.e. increasing grade); $83 \%$ of grade III tumours had metastases with moderate/strong CD44s expression, as compared to only $9 \%$ of grade I tumours $(\mathrm{p}=0.009)$ (Table II).

Disease-specific survival (DSS) and disease-free survival (DFS) were calculated, based on the time from diagnosis to death (due to disease), and on the time from diagnosis to the appearance of metastatic disease, respectively. In calculating DSS, all patients who died of other or unknown causes were treated as censored cases. DFS was shorter among (Stage B and C) patients whose metastases showed moderate/strong CD44s expression, 8.3 months compared to 16.9 months in patients with negative/weak CD44s expression in their metastases ( $\mathrm{p}=0.221$, Mann-Whitney test). In this analysis, all Dukes D cases were omitted, because they already had metastases at the time of diagnosis. No such association was found for CD44v6.

There was no significant association of CD44s or CD44v6 expression with age, sex and other clinical or histopathological variables including, tumour localization, TNM staging and CEA levels. Similarly, CD44s and CD44v6 in the primary tumours were not predictors of DFS or DSS in univariate or multivariate analyses.

\section{Discussion}

This is the first study, where CD44 expression levels in primary colorectal cancer are compared with their corresponding metastases, and showing that CD44 expression in the metastatic lesions is closely related to the advanced stage of the disease, a high grade of the primary tumour, and a short time to relapse. These data contribute to a better understanding of the events predisposing to development of the metastatic phenotype in CRC, which is essential to help identify the patients at increased risk for metastasis, to be subjected to closer surveillance and individually tailored therapies.

In the metastatic process of all epithelial neoplasia, loss of adhesive functions and gain of new adhesive functions are thought to play a critical role (17). CD44 is one of the molecules suggested to play a role in the development of metastases in the lymph nodes and in distal organs (18). In normal colon, members of the CD44 family are weakly expressed by a few epithelial cells at the base of crypts (13). The exact role of CD44s in epithelial metastases is more controversial, however, some studies suggest that CD44 up-regulation might be important in tumour invasion and spread of malignancies $(19,20)$, while others implicate that down-regulation or absence of CD44 is a reflection of tumour aggressiveness $(21,22)$. There is also some debate concerning the significance of the CD44v6 isoform in CRC, with conflicting reports regarding its prognostic value. While several authors report that $\mathrm{CD} 44 \mathrm{v} 6$ expression is associated with the advanced stage of the disease, liver metastasis and poor prognosis in CRC (23-26), there are others who failed to find any significant correlation between CD44v6 expression and disease progression (27-29). As a continuation to our previous study disclosing up-regulated CD44v6 as an important predictor of treatment response in advanced CRC (16), the present study 
compared CD44s and CD44v6 expression in primary CRCs and their metastases, in order to cast further light on these controversies.

We found that the expression of both CD44s and CD44v6 variant was significantly higher (moderate/strong) in primary tumours as compared to their metastases (Fig. 2). Considering the fact that CD44s and CD44v6 expression is almost absent in normal colonic mucosa, this would implicate a considerable increase in expression during tumorigenesis. As compared to the primary tumours, metastatic lesions in general tended to show reduced or absent expression, suggesting that CD44s and CD44v6 expression is down-regulated during the phase of tumour dissemination and/or establishment of metastatic deposits at the specific organ sites. This reduction is only relative, however, and all metastatic lesions still expressing CD44s and CD44v6 at a high level as compared with normal colonic mucosa. This relative reduction of CD44 expression could signify that: i) it might confer a greater metastatic potential (by decreasing the cell adhesion) to the tumour cells during the dissemination process, or ii) these observed differences in CD44 expression might not occur until the cells have established a viable colony at the site of metastasis. Certainly, many genes that have been implicated in the process of metastasis are reported to be active at the final stage of tumour cell colonization $(30,31)$. However, if this were the case organ-specific differences in CD44 expression in the metastatic deposits would be expected, but in the present study, CD44s and CD44v6 expression did not correlate with the site of metastasis (data not shown in Tables).

The expression of CD44s (but not CD44v6) in the metastatic lesions was closely associated with both the grade of the primary tumour $(\mathrm{G})$, as well as with the depth of tumour invasion (T), both suggesting that the level of expression of CD44s in metastases may reflect the aggressiveness of the primary tumour of origin. The local invasion of the primary tumour was most advanced among the cases with moderate/ strong CD44s expression in metastases. This is consistent with the study of Fernandez et al who demonstrated a significant relationship between CD44s expression and the high proliferation rate and aggressiveness in colorectal tumours (32). However, other groups have reported that loss of differentiation is associated with the down-regulation of these adhesion molecules in adeno-squamous carcinomas (33). It is important to emphasise that the majority of these previous studies have examined CD44 expression in the primary tumours only, whereas we analysed both primary tumours and their metastases, which offers a better opportunity to monitor the dynamic changes in CD44 expression during the development of a metastatic phenotype.

The cell adhesion mechanisms play an important role in tumour invasion, including those mediating the attachment of tumour cells to extracellular matrix (ECM) and basement membranes (BM). Both the degradation of $\mathrm{BM}$ and migration through ECM are facilitated by the interaction of CD44 with hyaluronic acid. Therefore, it is possible that the overexpression of CD44s in the primary tumours provides the cells with an increased potential to migrate and invade ECM, and also facilitates their attachment at distant sites forming metastases that continue to express higher levels of CD44s. Indeed, the invasion of CRC has been shown to be dependent on the interaction between CD44s and ECM, via HA $(34,35)$. Consistent with this is our observation that the time elapsed for metastatic disease to develop was shorter in patients with strong/moderate expression of CD44s as compared to patients whose metastases showed negative/weak CD44s expression (8.3 months vs 16.9 months). However, this result was not significant because of the small number of patients $(n=18)$ that developed metastases during the follow-up (the majority of patients had metastatic disease at diagnosis and were excluded from this particular analysis). However, previous studies have reported a significant association of CD44s expression with the advanced stages of the disease and these studies showed that the expression of CD44 in CRC tissues is significantly higher than in adjacent normal tissues $(14,24)$ and also that the CD44 expression correlated with a poor prognosis in CRC $(24,36)$.

Failing to establish a direct prognostic value for CD44s or CD44v6 expression in the primary tumour suggests that, indeed, the changes in these expressions that take place in metastases may be more informative on the eventual disease outcome. As shown in this study, increased expression of CD44s and CD44v6 in the primary tumours facilitates local invasion and dissemination to distant metastatic sites. This is followed by a relative decrease in CD44s expression at the metastatic deposits, as indicated by the significant differences in CD44 expression between the primary tumour and its metastases. Indeed, when these primary-metastasis (P-M) pairs were treated as paired data using the Wilcoxon signed ranks test, the difference in both the CD44s and CD44v6 expression between primary tumours and their metastases was even more significant ( $\mathrm{p}=0.003 \mathrm{p}=0.0001)$, respectively.

Our finding that CD44s expression in the metastatic lesion may reflect the aggressiveness (i.e. stage and grade) of the primary tumour from which it has originated is an important novel observation and reveals an interesting link between the two lesions. We also observed that higher levels of CD44s expression in metastases were associated with a shorter DFS, which may not necessarily implicate a poor prognosis, if there is a good response to treatment, as previously shown to be the case with CD44v6 (16). This finding warrants further investigation to determine, whether we have identified a molecular marker related to the development of a metastatic phenotype in CRC.

In conclusion, the findings of this study suggest a potential role for CD44 family of adhesion molecules as a biological marker in CRC. This in turn could have important clinical implications.

\section{References}

1. Boyle P and Ferlay J: Cancer incidence and mortality in Europe, 2004. Ann Oncol 16: 481-488, 2005.

2. Cancer facts and figures. In: American Cancer Society, 2005.

3. Moertel CG, Fleming TR, Macdonald JS, Haller DG, Laurie JA, Goodman PJ, Ungerleider JS, Emerson WA, Tormey DC, Glick JH, et al: Levamisole and fluorouracil for adjuvant therapy of resected colon carcinoma. N Engl J Med 322: 352-358, 1990.

4. Staib L, Link KH, Blatz A and Beger HG: Surgery of colorectal cancer: surgical morbidity and five- and ten-year results in 2400 patients - monoinstitutional experience. World J Surg 26: 59-66, 2002. 
5. Midgley R and Kerr D: Conventional cytotoxic and novel therapeutic concepts in colorectal cancer. Expert Opin Investig Drugs 10: 1011-1019, 2001

6. Screaton GR, Bell MV, Jackson DG, Cornelis FB, Gerth U and Bell JI: Genomic structure of DNA encoding the lymphocyte homing receptor CD44 reveals at least 12 alternatively spliced exons. Proc Natl Acad Sci USA 89: 12160-12164, 1992.

7. Jalkanen S, Bargatze RF, de los Toyos J and Butcher EC: Lymphocyte recognition of high endothelium: antibodies to distinct epitopes of an 85-95-kD glycoprotein antigen differentially inhibit lymphocyte binding to lymph node, mucosal, or synovial endothelial cells. J Cell Biol 105: 983-990, 1987.

8. Naor D, Nedvetzki S, Golan I, Melnik L and Faitelson Y: CD44 in cancer. Crit Rev Clin Lab Sci 39: 527-579, 2002.

9. Ponta H, Wainwright D and Herrlich P: The CD44 protein family. Int J Biochem Cell Biol 30: 299-305, 1998.

10. Isacke CM and Yarwood $\mathrm{H}$ : The hyaluronan receptor, CD44. Int J Biochem Cell Biol 34: 718-721, 2002.

11. Lindblom A and Liljegren A: Tumour markers in malignancies (Review). BMJ 320: 424-427, 2000.

12. Jackson PA, Green MA, Pouli A, Hubbard R, Marks CG and Cook MG: Relation between stage, grade, proliferation, and expression of p53 and CD44 in adenomas and carcinomas of the colorectum. J Clin Pathol 48: 1098-1101, 1995.

13. Herrlich P, Pals S and Ponta H: CD44 in colon cancer. Eur J Cancer 31A: 1110-1112, 1995.

14. Khoursheed M, Mathew TC, Makar RR, Sonia L, Abul H, Asfar S, Al-Sayer H, Dashti HM and Al-Bader A: Expression of CD44s in human colorectal cancer. Pathol Oncol Res 8: 170-174, 2002.

15. Maula S, Huuhtanen RL, Blomqvist CP, Wiklund TA, Laurila P and Ristamaki R: The adhesion molecule CD44v6 is associated with a high risk for local recurrence in adult soft tissue sarcomas. Br J Cancer 84: 244-252, 2001.

16. Bendardaf R, Lamlum H, Ristamaki R and Pyrhonen S: CD44 variant 6 expression predicts response to treatment in advanced colorectal cancer. Oncol Rep 11: 41-45, 2004.

17. Hart IR, Goode NT and Wilson RE: Molecular aspects of the metastatic cascade. Biochim Biophys Acta 989: 65-84, 1989.

18. Dalchau R, Kirkley J and Fabre JW: Monoclonal antibody to a human leukocyte-specific membrane glycoprotein probably homologous to the leukocyte-common (L-C) antigen of the rat. Eur J Immunol 10: 737-744, 1980.

19. Joensuu H, Ristamaki R, Klemi PJ and Jalkanen S: Lymphocyte homing receptor (CD44) expression is associated with poor prognosis in gastrointestinal lymphoma. Br J Cancer 68: 428-432, 1993.

20. Joensuu H, Klemi PJ, Toikkanen S and Jalkanen S: Glycoprotein CD44 expression and its association with survival in breast cancer. Am J Pathol 143: 867-874, 1993.

21. Heider KH, Hofmann M, Hors E, van den Berg F, Ponta H, Herrlich P and Pals ST: A human homologue of the rat metastasisassociated variant of CD44 is expressed in colorectal carcinomas and adenomatous polyps. J Cell Biol 120: 227-233, 1993.

22. Kuppner MC, Van Meir E, Gauthier T, Hamou MF and de Tribolet N: Differential expression of the CD44 molecule in human brain tumours. Int J Cancer 50: 572-577, 1992.
23. Mulder JW, Wielenga VJ, Polak MM, van den Berg FM, Adolf GR, Herrlich P, Pals ST and Offerhaus GJ: Expression of mutant p53 protein and CD44 variant proteins in colorectal tumorigenesis. Gut 36: 76-80, 1995.

24. Ropponen KM, Eskelinen MJ, Lipponen PK, Alhava E and Kosma VM: Expression of CD44 and variant proteins in human colorectal cancer and its relevance for prognosis. Scand J Gastroenterol 33: 301-309, 1998.

25. Mulder JW, Kruyt PM, Sewnath M, Oosting J, Seldenrijk CA, Weidema WF, Offerhaus GJ and Pals ST: Colorectal cancer prognosis and expression of exon-v6-containing CD44 proteins. Lancet 344: 1470-1472, 1994.

26. Streit M, Schmidt R, Hilgenfeld RU, Thiel E and Kreuser ED: Adhesion receptors in malignant transformation and dissemination of gastrointestinal tumors. J Mol Med 74: 253-268, 1996.

27. Koretz K, Moller P, Lehnert T, Hinz U, Otto HF and Herfarth C: Effect of CD44v6 on survival in colorectal carcinoma. Lancet 345: 327-328, 1995.

28. Gotley DC, Fawcett J, Walsh MD, Reeder JA, Simmons DL and Antalis TM: Alternatively spliced variants of the cell adhesion molecule CD44 and tumour progression in colorectal cancer. Br J Cancer 74: 342-351, 1996.

29. Coppola D, Hyacinthe M, Fu L, Cantor AB, Karl R, Marcet J, Cooper DL, Nicosia SV and Cooper HS: CD44V6 expression in human colorectal carcinoma. Hum Pathol 29: 627-635, 1998.

30. Chekmareva MA, Kadkhodaian MM, Hollowell CM, Kim H, Yoshida BA, Luu HH, Stadler WM and Rinker-Schaeffer CW: Chromosome 17-mediated dormancy of AT6.1 prostate cancer micrometastases. Cancer Res 58: 4963-4969, 1998.

31. Goldberg SF, Harms JF, Quon K and Welch DR: Metastasissuppressed C8161 melanoma cells arrest in lung but fail to proliferate. Clin Exp Metastasis 17: 601-607, 1999.

32. Fernandez JC, Vizoso FJ, Corte MD, Gava RR, Corte MG, Suarez JP, Garcia-Muniz JL and Garcia-Moran M: CD44s expression in resectable colorectal carcinomas and surrounding mucosa. Cancer Invest 22: 878-885, 2004.

33. Ylagan LR, Scholes J and Demopoulos R: Cd44: a marker of squamous differentiation in adenosquamous neoplasms. Arch Pathol Lab Med 124: 212-215, 2000.

34. Kim HR, Wheeler MA, Wilson CM, Iida J, Eng D, Simpson MA, McCarthy JB and Bullard KM: Hyaluronan facilitates invasion of colon carcinoma cells in vitro via interaction with CD44. Cancer Res 64: 4569-4576, 2004.

35. Bullard KM, Kim HR, Wheeler MA, Wilson CM, Neudauer CL, Simpson MA and McCarthy JB: Hyaluronan synthase-3 is upregulated in metastatic colon carcinoma cells and manipulation of expression alters matrix retention and cellular growth. Int $\mathbf{J}$ Cancer 107: 739-746, 2003.

36. Visca P, Del Nonno F, Botti C, Marandino F, Sebastiani V, Di Tondo U, Donnorso RP, Trombetta G, Filippi S and Alo PL: Role and prognostic significance of CD44s expression in colorectal cancer. Anticancer Res 22: 2671-2675, 2002. 